\title{
Factibilidade da gastrectomia vertical aberta no Sistema Único de Saúde.
}

\section{Factibility of open vertical gastrectomy in Brazil's Public Health System.}

Carolina Mantovani de Oliveira ${ }^{1} @$; André Tha Nassiz²; Alcides José Branco Filho, TCBC-PR²; Luis Sérgio Nassif, ACBC-PR²; Thaise de Araujo Wrubleski²; Alice Pavanatto Cavassola'; Roberta Vaz Pintan²

\section{R E S U M O}

\begin{abstract}
Objetivo: analisar as características clínicas e epidemiológicas, as complicações pós-operatórias e a perda de peso em pacientes submetidos à gastrectomia vertical por via convencional, em uma instituição de saúde do Sistema Único de Saúde (SUS). Métodos: estudo transversal, longitudinal, retrospectivo realizado a partir da coleta de dados de prontuários para análise de variáveis em pacientes submetidos à gastrectomia vertical aberta, no SUS, no período de julho de 2013 a janeiro de 2017. Resultados: foram analisados 296 pacientes operados no período do estudo, dos quais $54 \%$ eram do sexo masculino; a média de idade foi de 39,9 anos $\pm 11,4$; o índice de massa corporal (IMC) médio no pré-operatório foi de $43,5 \mathrm{~kg} / \mathrm{m}^{2}$ e no pós-operatório, de $30,3 \mathrm{~kg} / \mathrm{m}^{2}$; a perda de excesso de peso foi de $73,6 \% ; 83,24 \%$ apresentaram uma perda de excesso de peso maior do que 50\%; o IMC pré-operatório foi maior no grupo com perda de peso menor do que $50 \%$. Observou-se uma taxa de complicações precoces com necessidade de internamento de 5,4\% e um índice de mortalidade de 1\%. Conclusão: a gastrectomia vertical aberta é uma técnica segura e eficaz para a perda de peso e que pode ser mais realizada no SUS. Dentre as variáveis avaliadas, o IMC prévio foi a única relacionada com o sucesso pós-operatório.
\end{abstract}

Descritores: Obesidade. Cirurgia Bariátrica. Gastrectomia. Complicações Pós-Operatórias. Sistema Único de Saúde.

\section{INTRODUÇÃO}

A tualmente, a obesidade é considerada um dos principais problemas de saúde pública mundial. É uma condição crônica que pode se tornar fator de risco para outras doenças, como hipertensão arterial sistêmica, diabetes mellitus, dislipidemia, esteatose hepática, doença do refluxo gastroesofágico e colelitíase ${ }^{1}$. Indivíduos com índice de massa corporal (IMC) igual ou superior a $25 \mathrm{~kg} / \mathrm{m}^{2}$ são considerados com excesso de peso, enquanto aqueles com IMC maior ou igual a $30 \mathrm{~kg} / \mathrm{m}^{2}$ têm obesidade. É uma doença multifatorial, recidivante e necessita de uma abordagem multiprofissional para sua prevenção e tratamento.

Segundo estatísticas da Organização Mundial da Saúde (OMS), um em cada nove adultos no mundo é obeso e a população acima do peso cresceu consideravelmente nas últimas décadas. Entre 1980 e 2013, a proporção mundial de adultos com IMC acima de $25 \mathrm{~kg} / \mathrm{m}^{2}$ subiu de $28,8 \%$ para $36,9 \%$ entre os homens, e de $29,8 \%$ para $38 \%$ entre as mulheres ${ }^{2}$. A prevalência de obesidade praticamente triplicou entre os anos de 1975 e $2016^{3}$.
No Brasil, $50 \%$ da população adulta e 15\% das crianças estão acima do peso, sendo que na região sul $56,08 \%$ dos adultos apresentam excesso de peso, e 24,6\% de indivíduos entre dez e 19 anos de idade e $35,9 \%$ das crianças entre cinco nove anos de idade estão acima do peso ideal ${ }^{4}$. Esse crescimento alarmante é refletido na grande procura por cirurgias bariátricas, ainda mais quando se considera que a perda de peso em longo prazo, através de tratamentos conservadores, como mudança nos hábitos de vida, entre as quais alimentação saudável e atividades físicas, falha em mais de $90 \%$ dos pacientes obesos.

Assim, a cirurgia bariátrica tem se mostrado uma alternativa eficaz e segura para a redução de peso. Em 2013, mais de 460 mil cirurgias bariátricas foram realizadas no mundo, sendo 150 mil no Canadá e nos Estados Unidos, e cerca de 86 mil no Brasil, onde o Sistema Único de Saúde (SUS) oferece este procedimento na rede pública desde o ano $2000^{5,6}$. Aqui, no entanto, o tempo na fila de espera para realizar a cirurgia pode chegar a três ou quatro anos.

1 - Pontifícia Universidade Católica do Paraná, Curso de Medicina, Curitiba, PR, Brasil. 2 - Hospital Santa Casa de Misericórdia, Serviço de Cirurgia Bariátrica e Metabólica, Curitiba, PR, Brasil. 
A técnica cirúrgica mais realizada era $\mathrm{O}$ Bypass Gástrico em Y-de-Roux (BGYR), mas, nos últimos anos, houve uma tendência maior para a realização da Gastrectomia Vertical $(G V)^{7}$, o que fez este procedimento se tornar o modelo mais empregado na América do Norte, representando aproximadamente 60\% das cirurgias em 2017. O BGYR, por outro lado, caiu de 36\%, no ano de 2011, para 17,8\%, em 2017, de acordo com estimativas da American Society for Metabolic and Bariatric Surgery (ASMBS) ${ }^{8}$. Contudo, no Brasil, o BYGR ainda se manteve como o procedimento mais comum ${ }^{9}$.

Efetividade, segurança e resolução das comorbidades têm tornado a GV uma cirurgia ímpar no tratamento da obesidade. Estudos apontam uma perda média de $50 \%$ a $70 \%$ do excesso de peso, como consequência da redução do apetite pela ressecção do fundo gástrico ${ }^{10}$. Com a manutenção fisiológica do fluxo do trato gastrointestinal, resultados desfavoráveis, como síndrome de dumping, dificuldades no diagnóstico de tumores no estômago excluso, disabsorção e complicações nutricionais graves, ficaram praticamente ausentes com esta técnica. A GV permite ainda que, se necessário, possam ser realizados novos procedimentos revisionais ou complementares. A melhora do diabetes mellitus e da hipertensão arterial também se equivalem às taxas do $B G Y R^{11}$. Alguns autores descrevem este procedimento como sendo o ideal para adolescentes que necessitam de tratamento cirúrgico para obesidade, uma vez que apresenta baixos índices de complicações quando comparado às outras técnicas cirúrgicas ${ }^{12}$.

Apesar do aumento da GV como procedimento bariátrico definitivo ao redor do mundo, esta não é a realidade no SUS: entre 2013 e 2018 foram realizadas 1.629 GV e 43.211 BGYR, ou seja, apenas 3,7\% das cirurgias bariátricas realizadas neste período foram $\mathrm{GV}^{13,14}$.
O objetivo deste trabalho é analisar características clínicas e epidemiológicas, complicações pósoperatórias e perda de peso em pacientes submetidos à GV por via convencional em uma instituição de saúde do SUS.

\section{MÉTODOS}

Estudo transversal, longitudinal, retrospectivo, de pacientes submetidos à GV por via convencional no Serviço de Cirurgia Bariátrica e Metabólica do Hospital Santa Casa de Misericórdia (HSCM) de Curitiba, no período de julho de 2013 a janeiro de 2017. Foram incluídos os pacientes que tiveram acompanhamento pós-operatório por ao menos seis meses. O trabalho foi submetido e aprovado pelo Comitê de Ética e Pesquisa (CAAE 79067317.0.0000.0020).

A coleta de dados foi feita por meio da análise de prontuários com registro de informações como sexo, idade, técnica operatória e IMC pré e póscirúrgico, em seguimento de um ano. Além disso, foram investigadas complicações pós-operatórias precoces e tardias, e óbitos. Todos os dados foram obtidos pelos pesquisadores e registrados no instrumento de coleta de dados.

Os resultados de variáveis quantitativas foram descritos por média, desvio padrão, valor mínimo e valor máximo. Variáveis categóricas foram descritas por frequência e percentual. A avaliação da perda de peso foi realizada por meio da perda percentual do excesso de peso (\%PEP) através da fórmula (IMC pré-operatório - IMC após um ano)/ (IMC pré-operatório - 25). Os pacientes foram divididos em dois grupos: grupo Sucesso da Cirurgia, com $\%$ PEP $>50 \%$, e grupo Fracasso da Cirurgia, com $\% \mathrm{PEP}<50 \%$. A taxa de sucesso da cirurgia, de acordo com a perda de peso, foi comparada com outras variáveis, como sexo, idade e IMC pré-operatório. 
Para a comparação dos dois grupos, em relação às variáveis quantitativas, foi usado o teste $t$ de Student para amostras independentes. Variáveis categóricas foram analisadas considerando-se o teste exato de Fisher. A condição de normalidade foi avaliada pelo teste de Kolmogorov-Smirnov. Valores de $p<0,05$ indicaram significância estatística. Os dados foram analisados com o programa computacional Stata/SE v.14.1. StataCorpLP, USA.

A técnica da GV por via convencional é padronizada no nosso Serviço e se inicia com incisão mediana sub-xifoide de aproximadamente 12 a $15 \mathrm{~cm}$. A ligadura dos grandes vasos da grande curvatura gástrica é realizada desde 3,0 a 5,0 cm do piloro até o ângulo de Hiss com uso de dispositivo de energia bipolar reutilizável e sua secção é feita com energia monopolar. O uso de clipes metálicos para ligadura de vasos curtos é empregada esporadicamente. Para o grampeamento vertical utilizamos grampeador linear GIA 80 e três a cinco cargas azuis no total. Nos casos em que o antro é muito espesso, optamos pelo uso de carga verde para o primeiro grampeamento. É rotina no Serviço uma sobressutura invaginante das linhas de grampo com uso de polidioxanona 3-0.

\section{RESULTADOS}

Foram estudados 296 pacientes. Destes, 197 (67\%) tinham prontuários completos que puderam ser utilizados para cálculo dos dados epidemiológicos, complicações e perda de peso.
Os outros 99 (33\%) pacientes apresentavam prontuários incompletos ou abandonaram o seguimento com menos de seis meses e suas informações foram utilizadas apenas para o cálculo de dados epidemiológicos e complicações precoces.

Houve um leve predomínio do sexo masculino (54\% versus $46 \%$ ). A idade dos pacientes foi, em média, de 39,9ะ11,4 anos, com uma variância de 16 a 78 anos (Tabela 1).

O IMC pré-operatório foi, em média, de $43,5 \mathrm{~kg} / \mathrm{m}^{2} \pm 5,9$ e, após um ano do procedimento cirúrgico, este IMC reduziu seus valores para $30,3 \mathrm{~kg} /$ $\mathrm{m}^{2} \pm 5,0$, ou seja, a redução do IMC em um ano obteve uma média de $13,1 \mathrm{~kg} / \mathrm{m}^{2} \pm 4,7$. A queda nos valores do IMC representou uma perda de excesso de peso (\%PEP) de 73,6\% $\pm 22,9$. A média de seguimento dos pacientes foi de 15,6 meses $\pm 10,5$. Dos 197 pacientes com dados necessários para análise, 85,3\% obtiveram sucesso cirúrgico, ou seja, apresentaram \%PEP $>50 \%$, e apenas $14,7 \%$ se mantiveram com valores de $\% \mathrm{PEP}<50 \%$.

A comparação entre os dados epidemiológicos dos grupos que obtiveram e não obtiveram sucesso cirúrgico pode ser vista na tabela 2 . A média de IMC do grupo que obteve sucesso com a cirurgia foi de $42,9 \mathrm{~kg} / \mathrm{m}^{2} \pm 5,4$ contra uma média de IMC de $46,4 \mathrm{~kg} / \mathrm{m}^{2} \pm 7,0$ do grupo com falha no emagrecimento $(p<0,003)$. Com a GV, $88,8 \%$ dos homens e $81,1 \%$ das mulheres obtiveram sucesso cirúrgico. Sexo e idade não se mostraram variantes estatisticamente significantes entre os dois grupos.

Tabela 1. Perfil epidemiológico dos pacientes submetidos à gastrectomia vertical e tempo de seguimento.

\begin{tabular}{|c|c|c|c|}
\hline Variável & $n$ & Classificação & Resultados* \\
\hline Idade (anos) & 296 & & $39,9 \pm 11,4(16,3-78,5)$ \\
\hline \multirow[t]{2}{*}{ Sexo } & 296 & Masculino & $158(53,4)$ \\
\hline & & Feminino & $138(46,6)$ \\
\hline Tempo de seguimento (meses) & 287 & & $15,6 \pm 10,5(0,1-102,2)$ \\
\hline
\end{tabular}

* Descrito por média \pm desvio padrão ou por frequência (percentual). 
Tabela 2. Comparação entre os dados epidemiológicos dos grupos que obtiveram e não obtiveram sucesso cirúrgico na perda de peso após gastrectomia vertical.

\begin{tabular}{lcccc}
\hline \multirow{2}{*}{ Variável } & Classificação & \multicolumn{2}{c}{ Sucesso } & \multirow{2}{*}{ * $^{*}$} \\
\cline { 3 - 4 } & & Não $(\mathrm{n}=29)$ & $\operatorname{Sim}(\mathrm{n}=169)$ & \\
\hline Idade (anos) & & $43,7 \pm 14,0(18,4-67)$ & $40,8 \pm 11,0(16,3-78,5)$ & 0,208 \\
IMC pré-operatório $\left(\mathrm{kg} / \mathrm{m}^{2}\right)$ & & $46,4 \pm 7,0(35-61,9)$ & $42,9 \pm 5,4(34,7-62,5)$ & 0,003 \\
Sexo & Masculino & $12(11,2 \%)$ & $95(88,8 \%)$ & 0,159 \\
& Feminino & $17(18,9 \%)$ & $73(81,1 \%)$ & \\
\hline
\end{tabular}

* Teste $t$ de Student para amostras independentes (idade e IMC), teste exato de Fisher (sexo); $p<0,05$.

Dezesseis $(5,4 \%)$ pacientes precisaram de reinternamento ou prolongamento do internamento primário por complicações precoces (até 60 dias da cirurgia). As causas destas complicações foram infecção de sítio cirúrgico, deiscência de ferida operatória, infecção urinária, sepse de foco pulmonar, trombose venosa profunda (TVP), tromboembolismo pulmonar (TEP) e deiscência do coto gástrico. Destes reinternamentos, 11 (3,7\%) pacientes precisaram ser reoperados, sendo que sete $(2,3 \%)$ foram por deiscência do coto gástrico. Todos os casos de deiscência do coto gástrico foram tratados inicialmente por videolaparoscopia para drenagem cirúrgica da cavidade, sendo que em quatro casos foi realizada jejunostomia e em três casos foi realizada passagem intraoperatória de cateter nasoenteral (CNE). A endoscopia digestiva alta foi necessária em muitos desses casos para reposicionamento do CNE ou dilatação de subestenose da incisura angularis. Dois desses pacientes desenvolveram fístula gastrocutânea crônica e foi necessária conversão para BGYR, sendo que um apresentou desfecho favorável e outro evoluiu para óbito. Os outros cinco pacientes com deiscência do coto gástrico apresentaram melhora completa dos sintomas. Foram contabilizados três óbitos por complicações precoces em nossa casuística, o que representou um índice de mortalidade de $1 \%$.

Dentre as complicações tardias (após 60 dias da cirurgia), as mais prevalentes foram doença do refluxo gastroesofágico, colelitíase e hérnia incisional.

\section{DISCUSSÃO}

No Brasil, entre julho de 2013 e janeiro de 2017, período do nosso estudo, foram realizadas 27.409 cirurgias bariátricas pelo SUS, sendo que 1.384 foram GV e o restante BGYR ${ }^{15}$. Assim, nossa casuística de 296 GV realizadas no HSCM de Curitiba representou $21,3 \%$ do volume nacional.

O grupo de cirurgiões do presente estudo tende a indicar mais a técnica de GV para homens, por acreditar que estes apresentam taxa de perda de peso maior devido a um hábito alimentar mais quantitativo, associado ao estilo de vida sedentário. Estudos mostram que os homens procuram a cirurgia bariátrica quando a obesidade afeta suas atividades rotineiras ${ }^{16}$. A GV tende a ser mais indicada para esses pacientes, uma vez que o componente restritivo permite maior perda de peso em menor tempo. Já, as mulheres, que mais fazem este tipo de procedimento, procuram tratamento por motivação estética ${ }^{17,18}$. No presente estudo, a diferença entre as taxas de sucesso, de acordo com o sexo, não se mostrou estatisticamente significante. Além disso, não foi possível analisar o perfil alimentar dos pacientes envolvidos no estudo, fato que poderia contribuir para a elucidação das diferentes taxas de sucesso.

Em estudos prévios, foi demonstrado que a média de idade dos pacientes que realizam GV foi de 41,5 $\pm 11,9$ anos, em concordância com nossos resultados $^{18}$. Pela análise estatística, a idade do paciente não influenciou na perda de peso. 
A média do \%PEP nesse trabalho foi de $73,6 \%$, maior do que o observado em outro estudo brasileiro em que o seguimento após um ano de procedimento mostrou um resultado de $\%$ PEP de $67 \%{ }^{19}$. Um total de 168 pacientes, entre os 197 avaliados, obtiveram um \%PEP>50\%, representando $85,27 \%$ de sucesso cirúrgico. Quando comparamos nossos resultados com os de outros autores, observamos \%PEP>50\% em 94,7\% e $86,5 \%$ dos pacientes avaliados 20,21 .

Ao avaliar o IMC e \%PEP neste trabalho, foi possível observar que a média do IMC foi mais alta nos pacientes que não apresentaram sucesso cirúrgico quando comparado ao grupo com sucesso. Isso é condizente com múltiplos trabalhos na literatura que observaram menor perda de peso quanto maior o IMC inicial. Apesar da cirurgia bariátrica pela técnica de GV ser um procedimento bem estabelecido dentro da especialidade, ainda não se sabe o exato motivo pelo qual alguns pacientes com obesidade mórbida não conseguem obter perda de peso adequada após a cirurgia. Alguns estudos enfatizam a relação entre o cirurgião e o paciente, e fazem um plano de tratamento individual, tanto no pré quanto no pós-operatório, que incluem visitas de acompanhamento, exercícios e acompanhamento nutricional ${ }^{22}$. A implementação de protocolos individualizados pode auxiliar no sucesso pós-operatório dos pacientes.

A GV é conhecida por apresentar menores taxas de complicações perioperatórias. A literatura apresenta um risco de mortalidade de $1 \%$ a $2 \%$, tendo como principal causa o tromboembolismo pulmonar $^{23}$. O presente estudo obteve dois casos de TEP que necessitaram de reinternamento, porém sem mortalidade. Uma revisão literária abrangente encontrou apenas duas mortes após um mês da cirurgia ${ }^{24}$. No presente estudo, três pacientes evoluíram para óbito e tiveram como causa fístula gastrocutânea crônica, trombose de veia porta e infecção de partes moles.
Estes três casos representam 1\% do total de casos, estando de acordo com a porcentagem encontrada na literatura, mostrando que a cirurgia por GV representa um procedimento cirúrgico seguro quanto à taxa de mortalidade ${ }^{24}$.

Uma das complicações da GV é a deiscência de sutura do coto gástrico, que ocorre principalmente no angulo de Hiss e está presente em 0,7\% a 5,3\% dos $\operatorname{casos}^{24}$, sendo essa uma das complicações mais temidas e que exige tratamento complexo e multidisciplinar ${ }^{25}$. Em alguns casos pode ser realizada conversão para BGYR ou utilização de prótese endoscópica ${ }^{25}$. A estenose da incisura angularis ocorre em até 4\% das operações, cujo tratamento consiste principalmente na dilatação endoscópica. Em casos de estenose grave ou torção de tubo gástrico pode ser necessário intervenção cirúrgica com conversão para BGYR²6.

Outras complicações tardias possíveis de ocorrer são as hérnias incisionais, doença do refluxo gastroesofágico, anemia e reganho de peso ${ }^{27}$, sendo que as mais prevalentes neste estudo foram refluxo gastroesofágico, colelitíase e hérnia incisional.

Acreditamos que a GV por via convencional tem sido menos utilizada no SUS devido a três fatores. Primeiramente, devido à dificuldade técnica de exposição do fundo gástrico durante a via laparotômica em pacientes obesos, que exige uma curva de aprendizado mais longa do que o BGYR. Além disso, os pacientes do SUS eleitos para a realização de cirurgia bariátrica são de um nível socioeconômico mais baixo e têm uma tendência a apresentar pior perfil alimentar qualitativo, o que é desfavorável para uma técnica puramente restritiva. Por fim, o seguimento curto oferecido pelo SUS, de apenas 18 meses, também seria um dos fatores para a indicação limitada de GV ${ }^{16}$.

O presente estudo tem no seu desenho retrospectivo a sua maior limitação. Além disso, o seguimento curto de, em média, 15 meses não possibilita a verificação de complicações tardias, como o reganho de peso. 
Apesar do estudo apresentar um $n$ de pacientes considerável, não foi possível correlacionar o sucesso com dados demográficos, como idade e sexo. Estudos comparativos entre técnicas de cirurgias redutoras são de suma importância para a implementação de novos protocolos cirúrgicos.
Novos estudos, com desenhos prospectivos e seguimentos mais longos devem ser realizados para comparações mais fidedignas e acompanhamento de complicações tardias.

Através do presente estudo, foi possível concluir que a GV aberta é uma técnica eficaz e segura para a perda de peso e que pode ser mais utilizada no SUS.

\section{A B S T R A C T}

Objective: to analyse clinical and epidemiological characteristics, postoperative complications, and weight loss in patients undergoing conventional vertical gastrectomy in a hospital under Brazil's Public Health System (SUS). Methods: crosssectional longitudinal retrospective study based on data collection from medical records for variable analysis in patients undergoing open vertical gastrectomy in SUS, from July 2013 to January 2017. Results: we analysed 296 patients operated on during the study period, of which 54\% were male. The average age was of 39.9 years \pm 11.4 ; the average body mass index (BMI) was of $43.5 \mathrm{~kg} / \mathrm{m}^{2}$ in the preoperative period and of $30.3 \mathrm{~kg} / \mathrm{m}^{2}$ in the postoperative period; and the excess weight loss was of $73.6 \%$ (83.24\% had an excess weight loss greater than 50\%). Preoperative BMI was higher in the group with weight loss below $50 \%$. We observed a $5.4 \%$ rate of early complications requiring hospitalization and a 1\% mortality rate. Conclusion: open vertical gastrectomy is a safe and effective weight loss technique, which can be more performed in SUS. Among the variables evaluated in our work, the previous BMI was the only one related to the postoperative success.

Keywords: Obesity. Bariatric Surgery. Gastrectomy. Postoperative Complications. Unified Health System.

\section{REFERÊNCIAS}

1. National Institute of Health. The practical guide identification, evaluation, and treatment of overweight and obesity in adults. Bethesda (MD): $\mathrm{NIH} ; 2000$.

2. Marie $\mathrm{Ng}$, Fleming $\mathrm{T}$, Roinson $\mathrm{M}$, Thomson $\mathrm{B}$, Graetz N, Margono C, et al. Global, regional and national prevalence of overweight and obesity in children and adults 1980-2013: a systematic analysis for the Global Burden of Disease Study 2013. Lancet. 2014;384(9945):766-81. Erratum in: Lancet. 2014;384(9945):746.

3. World Health Organization. Obesity and overweight. Geneva, Switzerland: World Health Organization; 2018.

4. Brasil. Ministério da Saúde. VIGITEL Brasil 2014. Vigilância de Fatores de Risco e Proteção para Doenças Crônicas por Inquérito Telefônico [Internet]. 2014 Apr [citado 2019 Mar 7]. Disponível em: http://www.abeso.org.br/atitudesaudavel/mapa-obesidade.
5. Angrisani L, Santonicola A, lovino $P$, Formisano G, Buchwald H, Scopinaro N. Bariatric Surgery Worldwide 2013. Obes Surg. 2015;25(10):1822-32.

6. Kelles SMB, Machado CJ, Barreto SM. Dez anos de cirurgia bariátrica no Brasil: mortalidade intrahospitalar em pacientes atendidos pelo sistema único de saúde ou por operadora da saúde suplementar. ABCD Arq Bras Cir Dig. 2014;27(4):261-7.

7. Buchwald H, Oien DM. Metabolic/bariatric surgery Worldwide 2011. Obes Surg. 2013;23(4):427-36.

8. American Society for Metabolic and Bariatric Surgery. Estimate of Bariatric Surgery Numbers, 2011-2017 [Internet]. 2018 Jun [cited 2019 Mar 7]. Available from: https://asmbs.org/resources/estimate-of-bariatricsurgery-numbers

9. Herrera MF, Valencia A, Cohen R. Bariatric/metabolic surgery in Latin America. Am J Gastroenterol. 2019;114(6):852-3.

10. Lemos SLS, Thiago Domingos A, Vinha JM, Nadai $A P$, Vasconcellos $C P$, Ferragut CB. Nova proposta de tratamento cirúrgico da obesidade: gastrectomia vertical e bypass intestinal parcial. Resultados preliminares. Rev Bras Videocir. 2005;3(3):131-42. 
11. Lee CM, Taller J, Feng JJ, Jossart GH, Cirangle PT. The best bariatric operation for older patients: outcome after the band, vertical gastrectomy, Rouxen-y gastric bypass, and duodenal switch. Digestive Disease Week and the 46th Annual Meeting of the Society of the Surgery of the Alimentary Tract, Chicago, Illinois, May 16th, 2005. Disponível em: http://www.lapsf.com/vertical-gastrectomyweightloss-surgery.php . Acesso em 04 out 2019.

12. Baltasar A, Serra C, Bou R, Bengochea M, Andreo L. Sleeve gastrectomy in a 10-year-old child. Obes Surg. 2008;18(6):733-6.

13. Keidar A, Appelbaum L, Schweiger C, Schweiger C, Elazary R, Baltasar A. Dilated upper sleeve can be associated with severe postoperative gastroesophageal dysmotility and reflux. Obes Surg. 2010;20(2):140-7.

14. Brasil. Ministério da Saúde. Sistema de Informações Hospitalares do SUS (SIH/SUS) [Internet]. Brasília (DF): DATASUS; c2013-2019 [citado 2019 Mar 14]. Disponível em: http://tabnet.datasus.gov.br/cgi/ deftohtm. exe?sih/cnv/qiuf.def

15. Castanha $C R$, Ferraz $A A B$, Castanha $A R$, Belo GQMB, Lacerda RMR, Vilar L, et al. Avaliação da qualidade de vida, perda de peso e comorbidades de pacientes submetidos à cirurgia bariátrica. Rev Col Bras Cir. 2018;45(3):e1864.

16. Driscoll S, Gregory DM, Fardy JM, Twells LK. Longterm health-related quality of life in bariatric surgery patients: a systematic review and meta-analysis. Obesity (Silver Spring). 2016;24(1):60-70.

17. Abu-ghanem $Y$, Meydan C, Segev L, Rubin M. Gastric wall thickness and the choice of linear staples in laparoscopic sleeve gastrectomy: challenging conventional concepts. Obes Surg. 2017;27(3):837-43.

18. Costa RC, Yamaguchi N, Santo MA, Riccioppo D, Pinto-Junior PE. Outcomes on quality of life, weight loss, and comorbidities after Roux-en- $Y$ gastric bypass. Arq Gastroenterol. 2014;51(3):165-70.

19. Rocha QS, Fortes RC. Perda ponderal após gastroplastia em $Y$ de Roux e importância do acompanhamento nutricional - uma revisão de literatura. Comun Ciênc Saúde. 2011;22(1):61-9.
20. Zilberstein B, Galväo Neto M, Ramos AC. O papel da cirurgia no tratamento da obesidade. RBM Rev Bras Med. 2002;59(4):258-64.

21. Silva CF, Cohen L, Sarmento LD, Rosa FM, Rosado EL, Carneiro JR, et al. Effects of long-term Roux en-Y gastric bypass on body weight and clinical metabolic comorbidities in bariatric surgery service of a university hospital. ABCD Arq Bras Cir Dig. 2016;29(Suppl 1):20-3.

22. Cottam S, Cottam D, Cottam S, Cottam A. Sleeve gastrectomy weight loss and the preoperative and postoperative predictors: a systematic review. Obes Surg. 2019;29(4):1388-96.

23. Ribeiro R. Bypass gástrico. Rev Port Cir. 2008;II(4):6978.

24. Burgos AM, Braghetto I, Csendes A, Maluenda F, Korn O, Yarmuch J, et al. Gastric leak after laparoscopic-sleeve gastrectomy for obesity. Obes Surg. 2009;19(12):1672-7.

25. Lalor PF, Tucker ON, Szomstein S, Rosenthal RJ. Complications after laparoscopic sleeve gastrectomy. Surg Obes Relat Dis. 2008;4(1):33-8.

26. Cottam D, Qureshi FG, Mattar SG, Sharma S, Holover S, Bonanomi G, et al. Laparoscopic sleeve gastrectomy as an initial weight-loss procedure for high-risk patients with morbid obesity. Surg Endosc. 2006;20(6):859-63.

27. Arias E, Martínez PR, Li VKM, Szomstein S, Rosenthal RJ. Mid-term follow-up after sleeve gastrectomy as a final approach for morbid obesity. Obes Surg. 2009;19(5):544-8.

Recebido em: 15/09/2019

Aceito para publicação em: 22/10/2019

Conflito de interesse: nenhum.

Fonte de financiamento: nenhuma.

\section{Endereço para correspondência:}

André Tha Nassif

E-mail: andre-nassif@hotmail.com carolinamantovani@live.com

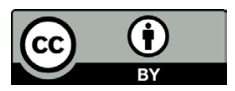

